\title{
SURGICAL TREATMENT OF CONGENITAL HEART DISEASE
}

\author{
By W. P. Cleland, M.R.C.P., F.R.C.S.
}

\section{Introduction}

The dramatic advance of cardiac surgery during the last decade was no chance or isolated affair, but was made possible by the many and varied advances in other fields of medicine and surgery. The history of cardiac surgery is littered with accounts of bold attempts to overcome surgical problems which were too often frustrated by lack of support in fields other than those of operative surgery. It is impossible to place the ancillary factors in any order of importance as they all play a very necessary role and each contributes some essential factor making for ultimate success.

Modern anaesthesia, providing as it does highly efficient ventilation with the use of relatively nontoxic agents which can be safely used for prolonged periods deserves, perhaps, pride of place. The better understanding of the effect of anaesthetic agents upon cardiac output and the peripheral circulation, the introduction of hypotensive and hypertensive agents, and the use of relaxants, have all helped to make modern cardiac anaesthesia remarkably safe and efficient.

Almost equally important has been the development of an efficient blood transfusion service which has ensured an adequate supply of blood at critical moments. This is, perhaps, of particular importance in the early developmental stages of a particular procedure when unexpected difficulties and complications may arise with alarming suddenness.

The employment of the many and varied antibiotics has played an important role in protecting vulnerable tissues from infection and has enabled these to be exposed and explored almost with impunity. The development of facilities for experimental surgery is an important feature in the growth of cardiac surgery which cannot be over emphasized. Such facilities are essential if advances are to be made, but in this country the facilities are poorly developed and adequate training in experimental surgery is difficult to obtain.

With the development of cardiac surgery the investigational side of cardiology has progressed considerably, especially in the fields of cardiac catheterization and angiocardiography. These investigations particularly provide valuable evidence about the anatomical deformities and the resultant physiological disturbances which are essential for the planning of surgical procedures.

\section{Historical Survey}

The first definite advance in the treatment of congenital cardiac disease came in 1939 with Gross's (1939) description of the ligation of the patent ductus arteriosus. In 1945, Crafoord (1945) and Gross (1945) independently described the first successful excision of the coarcted aorta with restoration of continuity of the vessel. A year later, Blalock and Taussig (1945) reported their important work on the treatment of Fallot's tetralogy. This work was of far-reaching importance and had a considerable effect upon future developments. Based upon careful physiological studies they introduced an entirely new principle into cardiac surgery, viz., the creation of a shunt between the systemic and pulmonary circulations whereby suboxygenated systemic blood was diverted to the oligaemic lungs for oxygenation. This work focused considerable attention on many of the problems of congenital heart disease and stimulated careful anatomical and physiological studies of these abnormalities by angiocardiography and cardiac catheterization. Surgical advances did not lag behind, and in the years following Blalock's work, many fundamental advances were made, in addition to many others which were mainly concerned with technical details.

The Blalock procedure is an extracardiac one and makes no attempt to correct any of the basic anatomical defects. In 1948, both Brock (1948) and Sellors (1948) described their attempts to relieve pulmonary stenosis by an direct attack on th pulmonary valve, employing an intracardiac procedure successfully for the first time in such cases.

The limitations of both the extracardiac and the closed intracardiac procedures soon became obvious when the more complicated abnormalities 
were investigated. Transposition of the great vessels and septal defects amongst others were not suitable for such methods and of recent years much research has been carried out on methods designed to provide conditions where direct open operations are possible on the heart. These procedures are still in the experimental stage, but promise well and will be discussed later.

\section{The Surgical Procedures}

At the present time the various cardiac operations can be grouped into extracardiac, closed intracardiac and open intracardiac procedures.

\section{Extracardiac Operations}

These procedures are largely carried out upon the great vessels entering or leaving the heart. The treatment of aortic aneurysm by aneurysmorrhaphy or excision and grafting, the relief of aortic coarctation, the ligation of the patent ductus, the treatment of vascular rings causing dysphagia and stridor (dysphagia lusoria) (Gross, 1946), are all examples. Perhaps the most important, from a fundamental point of view, has been the Blalock operation (subclavian pulmonary anastomosis), which is designed to increase the blood flow to the oligaemic lungs in Fallot's tetralogy. More recently, the transplantation of anomalous pulmonary veins and abnormal venae cavae have been carried out successfully.

These operations do not directly interfere with cardiac function but are, however, often long, tedious and exacting procedures, which often tell on both patient and surgeon.

\section{Closed Intracardiac Procedures}

These procedures are largely concerned with the relief of a valvular stenosis. The pulmonary valve was the first congenital lesion to be satisfactorily dealt with (Brock, 1948), although interest in the relief of mitral stenosis had been shown for many years. In the I920s, Cutler (1926), Graham (1922) and Souttar (1925) were all carrying out operations for the relief of mitral stenosis but the results were far from satisfactory and no further serious attempts were made until 1948 when Bailey (I949), Harken (I952) and Brock (1950) independently, and almost simultaneously, successfully relieved the obstruction of mitral stenosis. More recently, both aortic and tricuspid stenosis have been treated successfully.

Other closed intracardiac procedures include a variety of operations designed to close atrial septal defects of which the most satisfactory is the atrioseptopexy of Bailey (r954), in which the wall of the right atrium is sutured to the margins of the defect.

\section{Open Intracardiac Procedures}

The limitations of the extracardiac and the closed intracardiac procedures are very obvious and a great deal of experimental work has been $\_$ carried out in the last few years designed to provide conditions under which open cardiac operations can be carried out. Such an operation presents many formidable difficulties, of which the most important are:

(I) The survival of vital tissues (nervous system and cardiac muscle) during the period of circulatory arrest.

(2) The avoidance of cardiac irregularities.

(3) The avoidance of air embolism.

These difficulties have been partly overcome by one or other of the methods about to be described, but no one method has yet been evolved which does not carry certain disadvantages.

\section{Open Cardiac Operations}

The methods so far employed are:

I. Hypothermia. This consists of cooling the patient to temperatures of $26^{\circ}$ to $28^{\circ} \mathrm{C}$., at which os level it has been found that nervous tissue will survive a period of about ten minutes of complete circulatory arrest (Bigelow, I950; Swan, I954; and Ross, 1954). This period has enabled the $\frac{\mathbb{O}}{O}$ repair of atrial septal defects and the relief of pulmonary valvular and infundibular stenosis to be carried out satisfactorily. The time if $\vec{\theta}$ however, very limited and allows little margin fe coping with unexpected difficulties. The incidence of ventricular fibrillation is high althoug in many instances restitution of a normal rhythm is possible. Although hypothermia has been used with success in many instances, it is a procedure which has distinct limitations and disadvantages and in its present form cannot be regarded as entirely satisfactory.

2. Cross Circulation. This procedure has been developed by Lillehei (1954) and consists of the reciprocal transfer of blood from the arterial system of a donor to that of a recipient and from the venous system of the recipient back to that of the donor. A single pump is used in both circuits in order to preserve the balance between the circulations.

Completely compatible donors are, of course, necessary though in practice many near relatives are found to be suitable.

With this technique, Lillehei has found that the $N$ time during which the circulation through the $N$ heart can be arrested can be significantly pro- N longed and periods of up to 24 minutes have been $\stackrel{\omega}{\sigma}$ used without ill effect. Atrial and ventricular septal defects and pulmonary stenosis have been

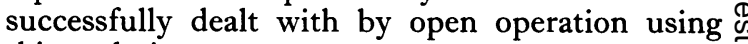
this technique.

There are obvious disadvantages about using a normal healthy individual in this way as a donor, 
although it is claimed that donor risks are negligible. However, there are undoubted moral considerations which make wider application of these methods uncertain.

3. Extra-corporeal Pump Oxygenator (HeartLung machine). A number of people throughout the world have been interested in the perfection of a completely isolated mechanical heart-lung unit which can be used to maintain a circulation whilst the heart and/or lungs are excluded from the circulation. Of the various machines, only three have proved satisfactory for clinical use (Gibbon, 1954; Melrose, 1953; and Crafoord, 1949). viz.:

In principle, the machines consist of three parts,

(a) A pump to remove blood from the venous system.

(b) An oxygenator.

(c) A pump to return blood to the arterial system.

The pump oxygenator can be used clinically in a variety of ways, e.g.:

(a) As a support to an already embarrassed heart which would enable operative procedures to be carried out with relative safety (e.g. Performance of aortic valvotomy in the very poor risk patient.) In such a case the circulation through the heart is maintained during the operation.

(b) As a complete cardio-pulmonary by-pass in order to permit open cardiac surgery on the empty heart.

(c) As a by-páss of either the left or the right side of the heart leaving the lungs in the circulation.

Clinical experience with the machine developed at the Postgraduate Medical School of London (Cleland and Melrose, 1955) has demonstrated its extreme efficiency as a pump and an oxygenator and no difficulty has been experienced in maintaining an adequate flow of oxygenated blood in the systemic vessels. Problems have arisen in the post-operative phase, however, from persistent and uncontrollable oozing into the chest and wound. These changes in the blood appear to be due to destruction of platelets and deposition of fibrinogen in the machine and should be capable of correction.

4. Controlled Cardiac Arrest. Melrose et al. (1955) have recently described a method of producing complete cardiac arrest by the use of intra-aortic injections of potassium citrate. This method appears to be highly accurate and absolutely controllable and repeatable and may have a considerable future in open cardiac operations particularly if used in conjunction with an extracorporeal circulation.

\section{Techniques in Cardiac Surgery \\ The Relief of Stenosis}

Congenital stenosis affects predominantly the pulmonary valve although both the aortic and the mitral valves are occasionally stenosed and capable of surgical relief. Coarctation of the aorta presents many of the basic problems of stenosis.

Obstruction of the aortic or pulmonary out-flow tracts may be either at the valve or in the infundibular region. The former is often a diaphragmatic dome and is readily amenable to instrumental incision and dilatation, using closed methods. Infundibular stenosis is produced by a localized or diffuse overgrowth of muscular tissue, in the sub-valvular region. Removal of the obstructing mass with ' punch forceps' by a closed technique has not proved entirely satisfactory and open methods are now preferred by some surgeons.

The effects of valvular obstruction can be readily determined. There is a raised pressure and muscular hypertrophy proximal to the obstruction and a low pressure and hypoplasia beyond the obstruction. The measurement of the pressure difference or gradient across the obstruction gives some indication of the severity of the lesion and determines whether it merits surgical treatment. Precise knowledge of the anatomy of the defect is desirable in the majority of cases but is often difficult to obtain. Careful catheter and angiocardiographic studies will often give important data.

Valvular obstruction can be approached in either an antero- or retrograde manner. The precise method employed will often depend upon such factors as ease of access, vulnerability and irritability of tissues, etc. Both the aortic and pulmonary valves are usually approached from the ventricle, although an approach through the aorta or pulmonary artery is preferred by some. The mitral and tricuspid valves are readily approached through the respective atria with remarkably little interference in cardiac function, but here again there are occasions when a retrograde approach through the ventricle is desirable.

Pulmonary Stenosis. This may be an isolated lesion (simple pulmonary stenosis) or one associated with other abnormalities (ventricular or atrial septal defect, Fallot's tetralogy, etc.). The lesion may be valvular or infundibular or a combination of the two.

All grades of severity are encountered in the simple group and the lesser degrees are compatible with a long and active life. The indications for operation depend largely on the degree of strain placed upon the right ventricle by the obstruction. This can be assessed clinically and measured by cardiac catheterization. Patients with right ventricular pressures of less than $50 \mathrm{~mm}$. Hg. do not 
require relief, whilst those with pressures above $100 \mathrm{~mm} . \mathrm{Hg}$. are in need of valvotomy. With pressure between $50 \mathrm{~mm}$. $\mathrm{Hg}$. and $100 \mathrm{~mm}$. $\mathrm{Hg}$. the indications for surgery are not clearly defined and these patients should be kept under observation if there are no other features indicating the need for surgery.

The main problem from the surgical point of view is to determine whether the stenosis is at the valve or below it. The presence of a dilated pulmonary artery (post-stenotic dilatation), of a thrill which arises in the valve but does not extend on to the ventricular aspect and the absence of an infundibular chamber (dilatation of the out-flow tract of the right ventricle immediately behind the valve which is seen in localized infundibular stenosis) all are in favour of a valvular stenosis. The actual size of the stenosis can often be determined by taking pressures from varying sites in the right ventricle and the pulmonary artery. Even so, it is sometimes very difficult to be certain about the precise anatomy of the lesion and especially so where combined lesions are present.

The problem will be largely solved if and when open procedures can be safely employed.

The pulmonary stenosis in Fallot's tetralogy can likewise be valvular or infundibular. In these cases we have the choice of a shunt operation or a direct valvotomy. If a solitary valvular stenosis is present, valvotomy is the treatment of choice, but otherwise a Blalock or Potts' operation is to be preferred.

Aortic Stenosis. Significant congenital aortic stenosis is not a common abnormality, although less important abnormalities of the valve (e.g. bicuspid) are more frequent. The obstructive lesions, like those of the pulmonary valve, may be valvular or subvalvular. The former may be diaphragmatic in type and is capable of surgical correction as described by Marquis and Logan (1955). Pressures on either side of the valve are, however, vastly different and on a much higher plane than is the case of the pulmonary valve and significant aortic incompetence is likely to follow over-enthusiastic valvotomy.

Coarctation of the Aorta. Narrowing of the aorta presents a very different set of problems from those posed by a narrowed valve. The operation field is remote from the heart and the latter is not interfered with appreciably. A very adequate collateral circulation is already established between the upper and lower halves of the body so that there is no particular danger attached to clamping the aorta even for considerable periods. A very careful meticulous mobilization of the aorta and its branches above and below the constriction is necessary to avoid damage to thin-walled tortuous and often aneurysmal branches. The aim of the surgeon is to excise the narrowed segment ando restore continuity by direct anastomosis without any residual constriction. The nearer the lumenz at the site of anastomosis approaches that of the $\stackrel{\mathbb{Q}}{\stackrel{\mathbb{Q}}{2}}$ aorta above and below the better is the resultc. likely to be. If the anastomotic lumen is less than 75 per cent. of that of the main trunk it is advisable? to employ a graft. Grafts may also be required? where longer segments of aorta have to be removed $\frac{\bar{\sigma}}{\bar{c}}$ for post-stenotic aneurysms or hypoplastic $\frac{\widetilde{\sigma}}{\widetilde{\alpha}}$ segments.

The risks of the operation are mainly those of haemorrhage from the suture line and the un- $-\overrightarrow{0}$ avoidable and unexpected accidents. The mortality rate is approximately 5 per cent. in experienced $\vec{\omega}$ hands so that one is completely justified in submit-? ting symptomless individuals to surgery at the present time especially as the prognosis of the untreated case is poor ( 95 per cent. of cases fail to survive to 45 years of age). The establishment of $\omega_{\mathcal{O}}$ a normal circulation in the lower limbs is the rule + but the blood pressure in the upper limbs fre-co quently remains above normal average levels윽 although it is inevitably reduced from its pre-operative level.

\section{Increase of Pulmonary Circulation}

In certain of the more complex congenitak abnormalities the flow of blood through the lungsco is hampered by stenosis at, or near the pulmonaty. valve, or by hypoplasia of the pulmonary arteg. Blood which normally should pass through thes lungs is diverted through a defect in one of the intracardiac septa from the right to the left side Blalock and Taussig (1945) first appreciated the importance of the defective pulmonary blood flow in cases of Fallot's tetralogy and were able to improve the flow by anastomosing a systemic artery to the pulmonary artery. The operation which bears his name (Blalock) consists of anas tomosing the right or left subclavian to the appropriate pulmonary artery. In this way, muchadditional sub-oxygenated systemic blood is passed through the lungs for oxygenation with relief of dyspnoea and cyanosis. Potts and Smith (1946) devised a modification of this procedureo whereby a shunt was created between the aorta and the left pulmonary artery at the approximate site of the obliterated ductus. This procedure is particularly applicable to small infants and in those cases where the subclavian is of insufficient length or size for a satisfactory Blalock operation.

A further modification of occasional value employs a preserved vessel graft to conduct blood from aorta to pulmonary artery.

\section{The Closure of Shunts}

Abnormal communications between the righto 
and left side of the heart or the pulmonary and systemic circulations are common.

Patency of the ductus arteriosus (common) and aorto-pulmonary septal defects (rare) are both examples of extracardiac shunts whilst ventricular and atrial septal defects produce an intracardiac shunt.

Closure of a patent ductus was the first of the congenital lesions to be treated successfully by surgical means (Gross, 1939), and is now a safe and satisfactory procedure with a low mortality. The duct is exposed as it lies above the left hilum and is either doubly ligated or divided and sutured.

The repair of aorto-pulmonary defects is more difficult largely because they are usually placed low down on the vessels near the valves and are usually very short and difficult to expose. Repair by open surgical methods would appear to be the only satisfactory means of dealing with the problem.

Unlike the closure of the patent ductus, the repair of ventricular and atrial defects is particularly difficult. Various closed methods of repairing atrial defects have been described and are effective in a number of instances. Of these the atrio-septopexy (Bailey, 1954) would appear to be the most satisfactory. In this operation, portion of the redundant wall of the right atrium is sutured to the margins of the defect with mattress sutures.

Sondergaard (1952) has employed an encircling suture which is so placed that it draws the margins of the defect together and has given promising results. This method has been further modified by Bjork and Crafoord (1953).

These closed techniques, however, have definite limitations, especially when large defects are present. This has led to the employment of open methods (Swan, r954), using hypothermia. This enables a careful exploration to be made and closure effected under direct vision and has much to recommend it in all cases. The technique involves the use of hypothermia which is still in an experimental stage of development although reasonably satisfactory techniques are now available.

Ventricular septal defects are technically more difficult to close; no satisfactory closed method has been developed and even with open methods there are many technical difficulties involved.

\section{Transposition of Anomalous Vessels}

All varieties of abnormally attached vessels are encountered in the medley of congenital abnormalities. Some are easy to correct without special facilities whilst others represent some of the most formidable problems in cardiac surgery.
Of the simpler varieties, two deserve especia mention:

(a) Abnormalities of the aortic arch or its branches which produce compression of the trachea and oesophagus (dysphagia lusoria). Such compression may produce severe and even fatal symptoms in young children or less frequently inlater adult life produce dysphagia or stridor dueto enlargement or rigidity associated with arterio $\frac{\bar{c}}{\mathrm{~S}}$ sclerotic changes in the vessels. Surgical cor $\frac{T}{8}$ rection is often readily performed with complete ${ }^{\triangle}$ relief of symptoms; the abnormal constrictingen vessel can often be anastomosed elsewhere, though $\overrightarrow{0}$ in many cases it may be safely ligated (Gross, I946).

(b) Abnormal drainage of the pulmonary veinsw into the right atrium may occur alone or ins conjunction with an atrial septal defect. The haemodynamics are similar to those of an atrial septal defect, which condition they will aggravate when the two are co-existent. Transposition of the veins is practicable in a number of cases. Abnormally entering venae cavae are less com- 6 monly encountered.

The more complex abnormalities are best represented by complete transposition of the great $_{\mathbb{P}}^{T}$ vessels. In these cases, reconstruction of the normal pathways can only be carried out withฏ complete arrest of the circulation through the heart and has not yet been carried out successfulf $\vec{c}$ in man, although it may well be practicable when open cardiac surgery has reached a higher level कै perfection.

\section{The Use of Grafts}

Artificial tubes (aortic homografts, plastic tubes, $\frac{0}{8}$ etc.) can be used either as a permanent pathway for $\overrightarrow{\vec{A}}$ the passage of blood (e.g. after excision of coarcta- $\frac{0}{3}$ tion) or as a temporary by-pass (shunt) employed to permit unhurried and accurate repair of part of the vascular system.

(a) Permanent Grafts. Aortic homografts pre- $\frac{\bar{\partial}}{3}$ served in special solution (Gross, 1949), or freeze dried (Eastcott, 1954), have been extensively employed during the past few years to bridge large $\delta$ defects in the aortic lumen following resection of complicated coarctations or aortic aneurysms. 웅 Homografts do not survive as such but act as an elastic scaffolding which is reinforced by the tissues of the host. The grafts have given satisfactory results when used in the thoracic aorta $ᄋ$ over a number of years, but the supply of suitable $N$ material presents considerable difficulties. N Recently plastic materials have been used with 0 success (nylon weave, orlon, terylene, polyvinyl alcohol sponge, etc.). These have the advantage $\frac{\odot}{\Phi}$ of adequate supply and ease of preparation to the correct size. They must, however, still be 0 regarded as being experimental. 
(b) Temporary Shunts. Stranahan (1955) and his associates have described ingenious methods of diverting blood from the aorta, using an aorta from a pig or calf in order to permit the resection of aneurysms of the arch.

Sarnoff (1955) has suggested diverting blood from the left ventricle into the thoracic aorta with the intention of approaching the aortic valve in a dry field.

These methods may have a definite application in the repair or resection of an aneurysm of the aortic arch and in aortic valve surgery although their sphere of usefulness will be limited as methods for open cardiac surgery develop.

\section{Artificial Valves}

Narrowing of the cardiac valves is capable of correction in a reasonably high proportion of cases. Incompetence is unfortunately a much more complex phenomenon and one which is vastly more difficult to treat surgically.

Many ingenious methods to reduce the leaking of mitral valves have been described (Bailey, 1954; Harken, 1954; and Logan, 1952), but none has proved lastingly satisfactory.

Hufnagel (1954) has employed a carefully prepared ball valve inserted into the thoracic aorta beyond the left subclavian artery in cases of severe incapacitating aortic incompetence and has claimed considerable improvement in the survivors.

It is obvious that the surgical treatment of incompetence has yet to be satisfactorily solved.

\section{Conclusion}

There seems little doubt that cardiac surgery is on the eve of important and far reaching advances dependent upon the development of facilities for unhurried surgical procedures on a dry and motionless heart. Various procedures are available at the present time but all carry certain risks and hazards which make widespread application unwise. Even when open methods of cardiac surgery can be safely employed it is probable that the well established closed procedures will still be extensively used and the more elaborate open methods reserved for the correction of the more complicated abnormalities.

\section{BIBLIOGRAPHY}

ALLEN, D. S. and GRAHAM, E. A. (1922), भ. Amer. med. Ass., 79,1028 .

BAILEY, C. P. (1949), Dis. Chest., 15, 377.

BAILEY, C. P., BOLTON, H. E., JAMISON, W. L. and NEPTUNE, W. B. (1953), F. Thor. Surg., 26, 184

, NICHOLS, H. T, and GERAEIN, A. E. BOLTON

Ibid., 28, 551 LS, H. T. and GERNEINHARDT, W. (1954),

BAKER,' C., BROCK, R. C. and CAMPBELL, M. (1950), Brit. med. $¥, 1,1283$.

BIGELOW, 'W. G., LINDSAY, W. K., HARRISON, R. C., GORDON, R. A. and GREENWOOD, W. F. (1950), Amer. F. Physiol., 160, 125.

BJOKK, V. O.' and CRAFOORD, 'C. (1953), f. Thor. Surg., 26, 300. $128,189$.

BROCK, R. C. (1948), Brit. med. F., i, 1121.

CLELAND, W. P. and MELROSE, D. G. (1955), Brit. med. Bull., ii, 236 .

CRAFOORD, C. and NYLIN, G. (1945), f. Thor. Surg., I4, 347

CRAFOORD, C. (1949), Surg. Gynae E Obstet., 89, 629.

CUTLER E. C. (1926)' Arch. Surg 12,212

EASTCOTT, H. H. G., HOLT, L. B., PEACOCK, J. H. and ROB, C. G. (1954), Lancet, i, 131.

GIBBON, J. H., MILLER., B., J. DOBELL, A. R., ENGELL, H. C. and voIGT, G. B. (1954), F. Thor. Surg., 28, 253.

GROSS, R. E. (1939), Ann. Surg., Iro, 321.

GROSS, R. E. (1945), Surgery, 18, 673 .

GROSS, R. E. (1946), Ann. Surg., 124, 532.

GROSS, R. E., BILL, A. H. and PIERCE, E. C. (1949), Surg.

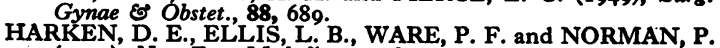
(1952), New Eng. Med. $\dot{F}_{\text {, }} 239,801$.

HARKEN,' D. E., BLACK, Ḧ., ELLIS, L. B. and DEXTER, L. (1954), $¥$. Thor. Surg., 28, 604

HUFNAGEL, C. A., HARVEY, W. P., RABIL, P. S. and MCDERMOTT, T. F. (1954), Surgery, 35, 673 .

LOGAN, A. and TÚRNER, R. (I052), Lancet 593 .

MARQUIS, R. M. and LOGAN, A. (I955), Br. Heart. Four., MELRO 373 .

MELROSE, D. G., DREYER, B., BENTALL, H. H. and BAKER, J. B. E. (1955), Lancet, ii, 21 .

POT'T, W. J., SMITH, S. and GIBSON, S. (1946), f.'Amer. med. Ass., 132, 627.

ROSS, D. N. (I954), Lancet, i, 1108.

SARNOFF, S. J., DONOVAN, T. J. and CASE, R. C. (1955), Circulation 9,564 .

SELLORS, T. H. (1948), Lancet, i, 988.

SONDERGAARD, T. (I952), Personal communication to Bjork and Crafoord, ' 7 . Thor. Surg., 26, 300.

STRANAHAN, A., ALLEY, R. D., SEWELL, W. H. and KANSEL, H. W.'. (1955), ๆ. Thor. Surg., 29, 54 .

KANSEL, H. W. (1955), $\mathcal{1}$. Thor. Surg., 29,
SWAN, H. (1953), F. Amer. med. Ass., 151, 792.

SWAN, H. (1953), f. Amer. med. Ass., 151, 792.
SWAN, H. and ZEAVIN, I. (1954), Ann. Surg., 139, 385.
WARDEN, H. E., COHEN, M., READ, R. C. and LILLEHEI C. W. (r954), $¥$. Thor. Surg., 28, 331 .

\section{RUTHIN CASTLE, NORTH WALES}

A Clinic for the diagnosis and treatment of Internal Diseases (except Mental or Infectious Diseases). The Clinic is provided with a staff of doctors, technicians and nurses.

The surroundings are beautiful. The climate is mild. There is central heating throughout. The annual rainfall is 30.5 inches, that is, less than the average for England.

The Fees are inclusive and vary according to the room occupied.

For particulars apply to THE SECRETARY, Ruthin Castle, North Wales. 